\title{
Effects of CrRu Underlayer and CrRu Capped Layer on the Microstructure and Magnetic Properties of FePt Films
}

\author{
S. C. Chen ${ }^{1}$, P. C. $\mathrm{Kuo}^{2}$, C. T. $\mathrm{Lee}^{2}$, A. C. $\mathrm{Sun}^{2}$, C. Y. $\mathrm{Chou}^{2}$, and Y. H. Fang ${ }^{2}$ \\ ${ }^{1}$ Department of Materials Engineering, MingChi University of Technology, Taipei 243, Taiwan, R.O.C. \\ ${ }^{2}$ Institute of Materials Science and Engineering, and Center for Nanostorage Research, National Taiwan University,
} Taipei 106, Taiwan, R.O.C.

\begin{abstract}
The magnetic properties and microstructure of $\mathrm{CrRu} / \mathrm{FePt}$ bilayer and $\mathrm{CrRu} / \mathrm{FePt} / \mathrm{CrRu}$ trilayer deposited by dc magnetron sputtering on preheated natural-oxidized $\mathrm{Si}(\mathbf{1 0 0})$ wafer substrates were studied. It is found that both the in-plane coercivity (Hc $/ /)$ and grain size of the FePt film increase with increasing the thickness of $\mathrm{CrRu}$ underlayer. The Hc// value of the FePt film is further increased but the grain size is decreased as adding a CrRu capped layer on the FePt film. The granular $L 1_{0}$ FePt nanoparticles with in-plane coercivity of 2300 Oe and isolated uniform size of $6.61 \mathrm{~nm}$ are achieved from the $\mathrm{CrRu}(15 \mathrm{~nm}) / \mathrm{FePt}(25 \mathrm{~nm}) / \mathrm{CrRu}(4 \mathrm{~nm})$ film deposited at a low substrate temperature of $350{ }^{\circ} \mathrm{C}$.
\end{abstract}

Index Terms-CrRu/FePt bilayer, $\mathrm{CrRu} / \mathrm{FePt} / \mathrm{CrRu}$ trilayer, in-plane coercivity, magnetron sputtering.

\section{INTRODUCTION}

$\mathbf{T}$ HE $L 1_{0} \mathrm{FePt}$ nanoparticles are a candidate material for next generation high-density magnetic recording media due to its excellent environmental stability and ultra high magnetocrystalline anisotropy $\left(\mathrm{Ku} \sim 7 \times 10^{7} \mathrm{erg} / \mathrm{cm}^{3}\right)$. However, $L 1_{0} \mathrm{FePt}$ phase usually develops at a higher temperature around $600{ }^{\circ} \mathrm{C}$ [1], [2], which results in the FePt nanoparticle agglomerate and grain growth. Therefore, the ordering temperature of $L 1_{0} \mathrm{FePt}$ phase must be reduced for preventing grain growth. In addition, the medium noise which caused by the magnetic grain interactions have to be also solved [3], [4].

Previous studies had reported that the grain size and magnetic grain interactions could be reduced by addition of various materials such as $\mathrm{Si}_{3} \mathrm{~N}_{4}$ [5] and AlN [6], but the addition of them would increase the ordering temperature of $L 1_{0}$ FePt. It has been known that the ordering temperature could be reduced by introducing a underlayer due to the lattice misfit between FePt film and underlayer [7], [8]. On the other hand, the deposition of capped layer on the FePt film could reduce exchange coupling between FePt magnetic grains [9], [10]. In this work, we use $\mathrm{CrRu}$ underlayer and capped layer to prepare $\mathrm{CrRu} / \mathrm{FePt}$ bilayer and $\mathrm{CrRu} / \mathrm{FePt} / \mathrm{CrRu}$ trilayer films, and investigate the effects of $\mathrm{CrRu}$ underlayer and $\mathrm{CrRu}$ capped layer on the grain size, saturation magnetization and coercivity of the FePt film.

Digital Object Identifier 10.1109/TMAG.2006.888487

Color versions of one or more of the figures in this paper are available online at http://ieeexplore.org.

\section{EXPERIMENT}

The $\mathrm{CrRu}$ underlayer thickness $\left(\mathrm{t}_{\mathrm{CrRu}}\right)$ in the range of 10-100 nm, the FePt magnetic layer of $25 \mathrm{~nm}$, and $4 \mathrm{~nm}$ $\mathrm{CrRu}$ capped layer are all deposited by dc magnetron sputtering at substrate temperature of $350{ }^{\circ} \mathrm{C}$. The base pressure in the sputtering chamber is better than $5 \times 10^{-7}$ Torr. The samples are cooled to room temperature under high vacuum in the sputtering chamber. The magnetic properties of the films are measured using a vibrating sample magnetometer (VSM) at room temperature. The microstructures of the films are investigated by a Philips Tecnai F30 field emission gun (FEG) transmission electron microscopy (TEM) and X-ray diffractometer (XRD) with $\mathrm{Cu}-\mathrm{K}_{\alpha}$ radiation. The compositions of the films are determined by EDS, and they are $\mathrm{Fe}_{55} \mathrm{Pt}_{45}$ and $\mathrm{Cr}_{90} \mathrm{Ru}_{10}$ for the FePt and $\mathrm{CrRu}$ films, respectively.

\section{RESULTS AND DisCUSSION}

Fig. 1 shows the variations of $\mathrm{Hc} / /$ and grain size of $\mathrm{FePt}$ films with varying $\mathrm{CrRu}$ underlayer thickness. Previous study [11] had reported that the in-plane coercivity of single layer FePt film with thickness below $30 \mathrm{~nm}$ only had several hundreds Oe after annealing at a low-temperature of $350{ }^{\circ} \mathrm{C}$. However, the $\mathrm{Hc}_{/ /}$value can be increased to 1700 Oe by introducing a $10 \mathrm{~nm}$ $\mathrm{CrRu}$ underlayer under the $25 \mathrm{~nm}$ FePt film which deposited at $350^{\circ} \mathrm{C}$, as shown in Fig. 1. And $\mathrm{Hc} / /$ is enhanced significantly to $4000 \mathrm{Oe}$ as $t_{\mathrm{CrRu}}$ is increased to $50 \mathrm{~nm}$. When the $t_{\mathrm{CrRu}}$ is further increased to $100 \mathrm{~nm}$, the $\mathrm{Hc} / /$ is further increased to 4280 Oe.

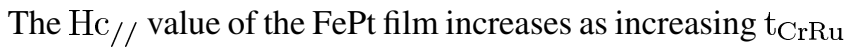
from 10 to $100 \mathrm{~nm}$ may be owing to the following reasons: 


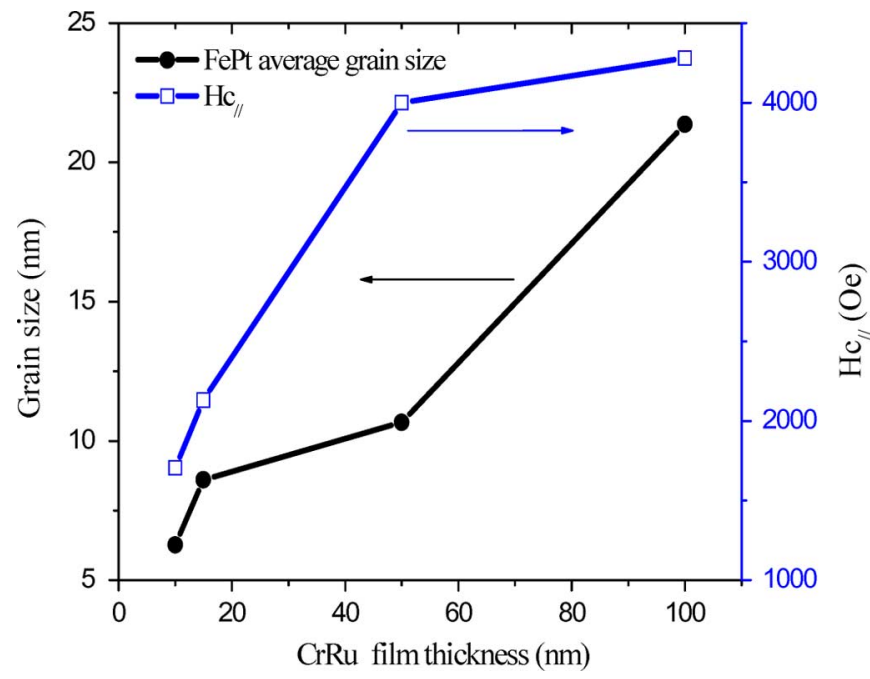

Fig. 1. The variations of $\mathrm{Hc}_{/ /}$and grain size of the FePt film with $\mathrm{CrRu}$ underlayer thickness of the $\mathrm{CrRu} / \mathrm{FePt}$ bilayer films which deposited at substrate temperature of $350{ }^{\circ} \mathrm{C}$.

(I) For randomly orientated noninteracting particles, the dependence of coercivity $(\mathrm{Hc})$ on the particle size (D) can be described as [12]

$$
\mathrm{Hc}=\frac{0.96 \mathrm{Ku}}{\mathrm{Ms}}\left[1-\left(\frac{D p}{D}\right)^{0.77}\right]
$$

where Ms is the saturation magnetization. The minimal stable grain size $(\mathrm{Dp})$ of FePt alloy is about $3 \mathrm{~nm}$ [13]. As shown in Fig. 1, the average grain size of FePt films increases from 6.3 to $21.3 \mathrm{~nm}$ as $\mathrm{CrRu}$ underlayer thickness increases from 10 to $100 \mathrm{~nm}$. According to above equation, the increase in particle size leads the increase in coercivity. (II) Wong et al. [14] had reported that the degree of interfacial misfit is larger as the grain size in $\mathrm{Cr} / \mathrm{Co}$ film is larger. Similarly, in our $\mathrm{CrRu} / \mathrm{FePt}$ system, the average grain size of $\mathrm{FePt}$ films is increased as increasing $t_{\mathrm{CrRu}}$ from 10 to $100 \mathrm{~nm}$ that results in the increase in degree of interfacial misfit in the $\mathrm{CrRu} / \mathrm{FePt}$ films, and the misfit defect in the FePt layer will be increased as the degree of interfacial misfit increases. This results that the transformation of soft $\gamma$-FePt to hard $L 1_{0}$ FePt phase becomes easier. Therefore, the coercivity of FePt film is increased due to the degree of order of FePt layer is increased, i.e., increase the amount of $L 1_{0} \mathrm{FePt}$ phase in the FePt layer as the $t_{\mathrm{CrRu}}$ is increased from 10 to $100 \mathrm{~nm}$.

Fig. 2 shows the M-H loops of (a) in-plane and (b) out-plane of the $\mathrm{CrRu}(15 \mathrm{~nm}) / \mathrm{FePt}(25 \mathrm{~nm})$ and $\mathrm{CrRu}(15 \mathrm{~nm}) / \mathrm{FePt}(25$ $\mathrm{nm}) / \mathrm{CrRu}(4 \mathrm{~nm})$ films. Comparing Fig. 2(a) with Fig. 2(b), we can see that both of the $\mathrm{CrRu} / \mathrm{FePt}$ and $\mathrm{CrRu} / \mathrm{FePt} / \mathrm{CrRu}$ films have excellent in-plane magnetic anisotropy. This is due to that the lattice misfit between $\mathrm{CrRu}(002)[100]$ and $\mathrm{FePt}(200)][110]$ is about $6.6 \%$ which leads the $L 1_{0} \mathrm{FePt}(200)$ plane can epitaxially grow along the $\mathrm{CrRu}(002)$ underlayer.

The Ms value of FePt films decreases from $730 \mathrm{emu} / \mathrm{cm}^{3}$ to $610 \mathrm{emu} / \mathrm{cm}^{3}$ as $4 \mathrm{~nm} \mathrm{CrRu}$ capped layer is added, as shown in Fig. 2(a). This may be due to the diffusion of $\mathrm{Cr}$ and $\mathrm{Ru}$ atoms from capped layer into $\mathrm{FePt}$ layer through grain boundary, and $\mathrm{Cr}$ or $\mathrm{Ru}$ atoms react partially with $\mathrm{Fe}$ or $\mathrm{Pt}$ atoms, which

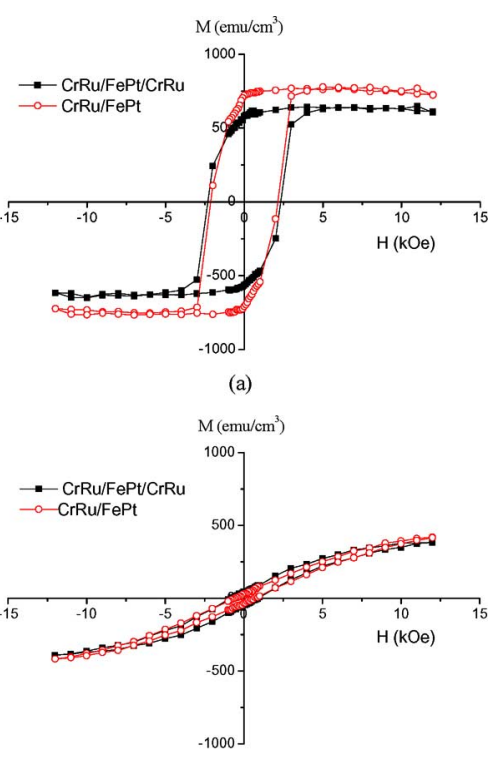

(b)

Fig. 2. The M-H loops of (a) in-plane (b) out-plane of the $\mathrm{CrRu}(15$ $\mathrm{nm}) / \mathrm{FePt}(25 \mathrm{~nm})$ bilayer and $\mathrm{CrRu}(15 \mathrm{~nm}) / \mathrm{FePt}(25 \mathrm{~nm}) / \mathrm{CrRu}(4 \mathrm{~nm})$ trilayer films which deposited at substrate temperature of $350{ }^{\circ} \mathrm{C}$.

results in decrease of Ms value. On the other hand, most of the $\mathrm{Cr}$ and $\mathrm{Ru}$ atoms are distributed at grain boundary of FePt that will increase the grain boundary energy and decrease the energy barrier of the transformation of $\mathrm{FePt}$ from soft $\gamma$-FePt to hard $L 1_{0} \mathrm{FePt}$ phase. Therefore, adding CrRu capped layer on the FePt film will also enhance the degree of order of FePt layer. The decrease in Ms value of $\mathrm{FePt}$ layer as adding $\mathrm{CrRu}$ capped layer may be also ascribed the increase of $L 1_{0} \mathrm{FePt}$ phase content in the film, because the Ms value of $L 1_{0} \mathrm{FePt}$ phase is lower than that of $\gamma$-FePt phase[15]. Increase the amount of $L 1_{0} \mathrm{FePt}$ phase in the FePt layer will enhance coercivity of $\mathrm{FePt}$ film, and therefore the $\mathrm{Hc} / /$ value of $\mathrm{FePt}$ films increases from $2130 \mathrm{Oe}$ to 2300 Oe when $4 \mathrm{~nm} \mathrm{CrRu}$ capped layer is added, as shown in Fig. 2(a).

Fig. 3 are the TEM bright field images and grain size distribution of (a) $\mathrm{CrRu}(15 \mathrm{~nm}) / \mathrm{FePt}(25 \mathrm{~nm})$ and (b) $\mathrm{CrRu}(15 \mathrm{~nm}) /$ $\mathrm{FePt}(25 \mathrm{~nm}) / \mathrm{CrRu}(4 \mathrm{~nm})$ films which deposited at substrate temperature of $350{ }^{\circ} \mathrm{C}$. It shows that the average grain size of the FePt film in $\mathrm{CrRu}(15 \mathrm{~nm}) / \mathrm{FePt}(25 \mathrm{~nm})$ bilayer is about 8.60 $\mathrm{nm}$ and the grain size distribution is large. When the $4 \mathrm{~nm} \mathrm{CrRu}$ capped layer is added, the average grain size decreases to 6.61 $\mathrm{nm}$ and the film morphology shows narrow size distribution and the particles are isolated, as shown in Fig. 3(b). Several studies had shown that reduction of grain size and increasing the grain size uniformity would decrease the medium noise of thin film media [3], [16].Therefore, the $\mathrm{Cr}$ and $\mathrm{Ru}$ atoms diffuse from capped layer into FePt layer can reduce grain growth and obtain isolated FePt particles with uniform size that is beneficial to reduce exchange coupling of the magnetic grains and decrease medium noise of FePt film.

\section{CONCLUSION}

Both the $\mathrm{Hc} / /$ value and grain size of the FePt film are increased as a $\mathrm{CrRu}$ underlayer is introduced. Further adding a 

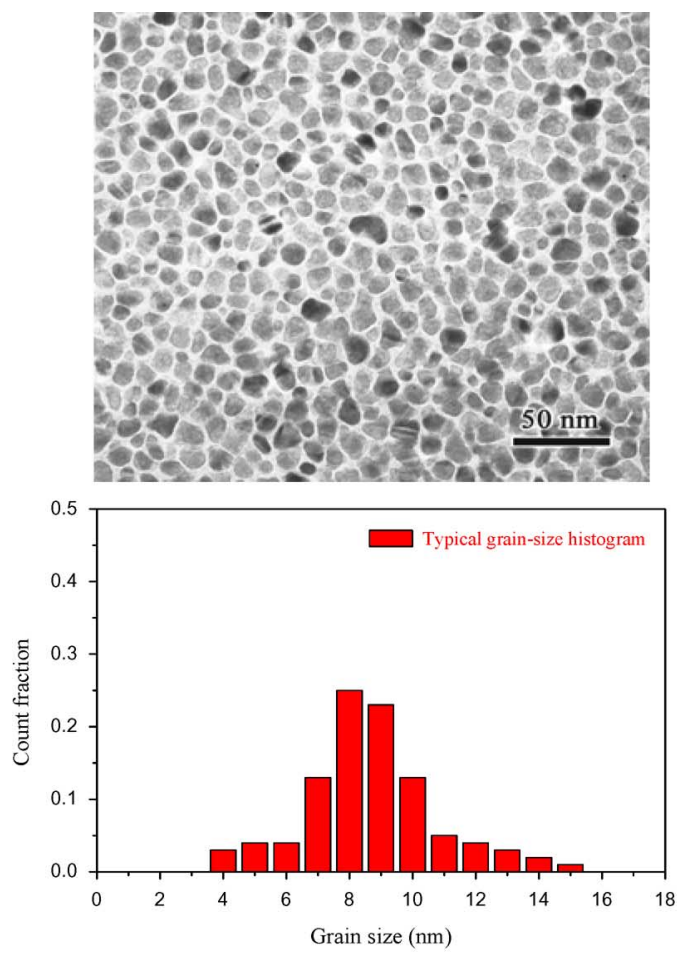

(a)
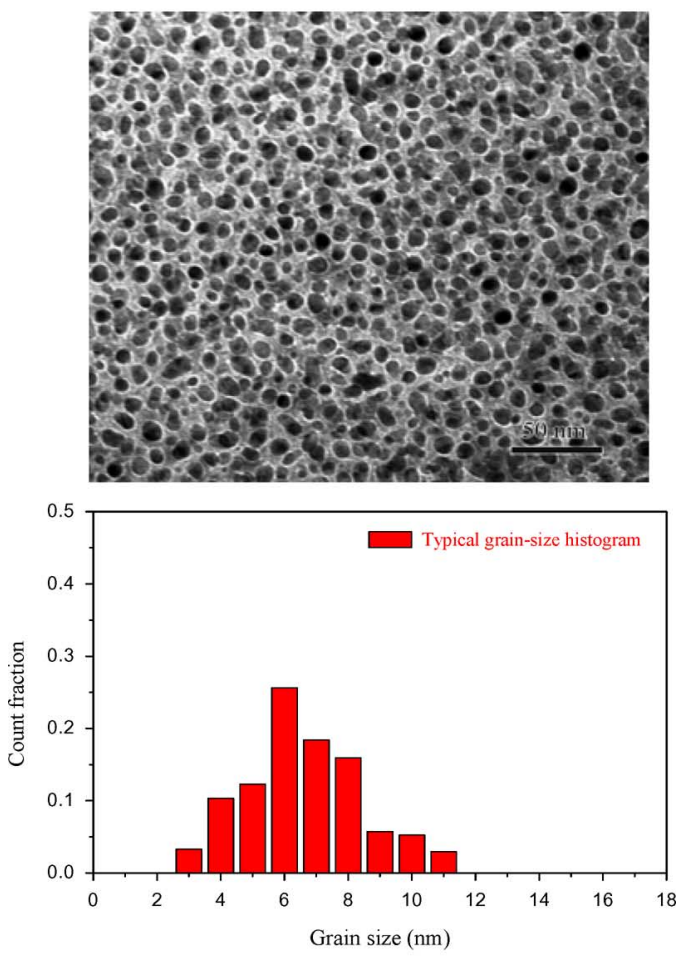

(b)

Fig. 3. The TEM bright field images and grain size distribution of (a) $\mathrm{CrRu}(15$ $\mathrm{nm}) / \mathrm{FePt}(25 \mathrm{~nm})$ bilayer and (b) $\mathrm{CrRu}(15 \mathrm{~nm}) / \mathrm{FePt}(25 \mathrm{~nm}) / \mathrm{CrRu}(4 \mathrm{~nm})$ trilayer films which deposited at substrate temperature of $350^{\circ} \mathrm{C}$.
CrRu capped layer onto FePt film, the isolated FePt grains with uniform grain size are obtained and the $\mathrm{Hc} / /$ of the FePt film is further increased, but the Ms value is decreased.

\section{ACKNOWLEDGMENT}

This work was supported by the National Science Council and Ministry of Economic Affairs of Taiwan through Grant NSC 94-2216-E-131-003 and 94-EC-17-A-08-S1-0006.

\section{REFERENCES}

[1] T. Suzuki and K. Ouchi, "Sputter-deposited (Fe-Pt)-MgO composite films for perpendicular recording media," IEEE Trans. Magn., vol. 37, pp. 1283-1285, 2001.

[2] P. C. Kuo, S. C. Chen, Y. D. Yao, A. C. Sun, and C. C. Chiang, "Microstructure and magnetic properties of nanocomposite FePtCr-SiN thin films," J. Appl. Phys., vol. 91, pp. 8638-8640, 2002.

[3] J. G. Zhu, "Transition noise properties in longitudinal thin film media," IEEE Trans. Magn., vol. 29, pp. 195-200, 1993

[4] Y. H. Lee, J. P. Wang, and L. Lu, "The role of amorphous $\mathrm{Ni}_{50} \mathrm{P}_{50}$ precoating layer in CoCrPtTa thin film media," J. Appl. Phys., vol. 87, pp. 6346-6348, 2000.

[5] C. M. Kuo and P. C. Kuo, "Magnetic properties and microstructure of FePt-Si ${ }_{3} \mathrm{~N}_{4}$ nanocomposite thin films," J. Appl. Phys., vol. 87, pp. 419-426, 2000.

[6] S. C. Chen, P. C. Kuo, C. T. Lie, and J. H. Hua, "Microstructure and coercivity of granular FePt-AlN thin films," J. Magn. Magn. Mater., vol. 236, pp. 151-157, 2001.

[7] Y. F. Ding, J. S. Chen, E. Liu, C. J. Sun, and G. M. Chow, "Effect of lattice mismatch on chemical ordering of epitaxial $L 1_{0}$ FePt films," $J$. Appl. Phys., vol. 97, p. 10H303, 2005.

[8] C. C. Chiang, C.-H. Lai, and Y. C. Wu, "Low-temperature ordering of $L 1_{0}$ FePt by PtMn underlayer," Appl. Phys. Lett., vol. 88, p. 152508, 2006.

[9] S. Jeong, T. Ohkubo, A. G. Roy, D. E. Laughlin, and M. E. McHenry, "In situ ordered polycrystalline FePt $L 1_{0}(001)$ nanostructured films and the effect of CrMn and $\mathrm{Zn}$ top layer diffusion," J. Appl. Phys., vol. 91, pp. 6863-6865, 2002.

[10] B. C. Lim, J. S. Chen, and J. H. Yin, "Reduction of exchange coupling and enhancement of coercivity of $L 1_{0} \mathrm{FePt}(001)$ films by $\mathrm{Cu}$ top layer diffusion," Thin Solid Films, vol. 505, pp. 81-84, 2006.

[11] A. C. Sun, P. C. Kuo, S. C. Chen, C. Y. Chou, H. L. Huang, and J. H. Hsu, "Magnetic properties and microstructure of low ordering temperature $L 1_{0}$ FePt thin films," J. Appl. Phys., vol. 95, pp. 7264-7266, 2004.

[12] D. H. Ping, M. Ohnuma, K. Hono, M. Watanabe, T. Iwasa, and T. Masumoto, "Microstructures of FePt-Al-O and FePt-Ag nanogranular thin films and their magnetic properties," J. Appl. Phys., vol. 90, pp. 4708-4716, 2001.

[13] D. Weller, A. Moser, L. Folks, M. E. Best, W. Lee, M. F. Toney, M. Schwickert, J.-U. Thiele, and M. F. Doerner, "High Ku materials approach to 100 Gbits/in ${ }^{2}$," IEEE Trans. Magn., vol. 36, pp. 10-15, 2000.

[14] B. Y. Wong, J. F. Ying, and K. Johnson, "Modeling of misfit induced defects in $\mathrm{Co} / \mathrm{Cr}$ recording media," IEEE Trans. Magn., vol. 36, pp. 2360-2362, 2000.

[15] T. Katayama, T. Sugimoto, Y. Suzuki, M. Hashimoto, P. dc Haan, and J. C. Lodder, "Magneto-optical Kerr rotation spectra in ordered and disordered phase of Fe-Pt alloy films," J. Magn. Magn. Mater., vol. 104-107, pp. 1002-1004, 1992.

[16] I. Kaitsu, A. Inomata, I. Okamoto, and M. Shinohara, "Magnetic and $\mathrm{R} / \mathrm{W}$ properties of $\mathrm{CoPt}-\mathrm{SiO}_{2}$ granular media," IEEE Trans. Magn., vol. 34, pp. 1591-1593, 1998.

Manuscript received August 10, 2006 (e-mail: sscchh@ms28.hinet.net). 